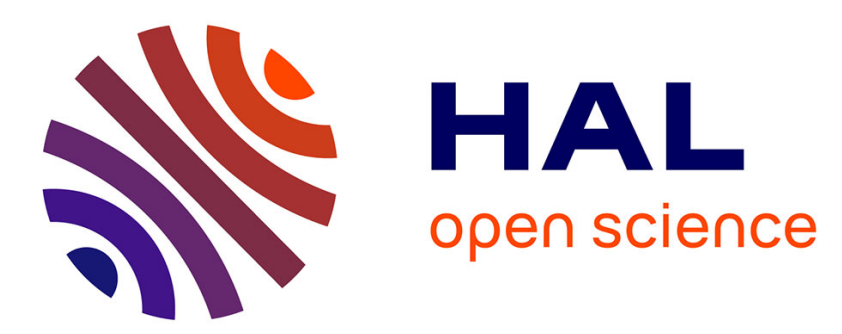

\title{
ISS Lyapunov functions for time-varying hyperbolic partial differential equations
}

Christophe Prieur, Frédéric Mazenc

\section{To cite this version:}

Christophe Prieur, Frédéric Mazenc. ISS Lyapunov functions for time-varying hyperbolic partial differential equations. CDC 2011 - ECC 2011 - 50th IEEE Conference on Decision and Control and European Control Conference, Dec 2011, Orlando, Floride, United States. pp.n.c., 10.1109/cdc.2011.6160401 . hal-00664201

\section{HAL Id: hal-00664201 \\ https://hal.science/hal-00664201}

Submitted on 30 Jan 2012

HAL is a multi-disciplinary open access archive for the deposit and dissemination of scientific research documents, whether they are published or not. The documents may come from teaching and research institutions in France or abroad, or from public or private research centers.
L'archive ouverte pluridisciplinaire HAL, est destinée au dépôt et à la diffusion de documents scientifiques de niveau recherche, publiés ou non, émanant des établissements d'enseignement et de recherche français ou étrangers, des laboratoires publics ou privés. 


\title{
ISS Lyapunov functions for time-varying hyperbolic partial differential equations
}

\author{
Christophe Prieur and Frédéric Mazenc
}

\begin{abstract}
A family of time-varying hyperbolic systems of balance laws is considered. The partial differential equations of this family can be stabilized by selecting suitable boundary conditions. For the stabilized systems, the classical technique of construction of Lyapunov functions provides with a function which is a weak Lyapunov function in some cases, but is not in others. We transform this function through a strictification approach which gives a time-varying strict Lyapunov function which allows us to establish asymptotic stability in the general case and a robustness property with respect to additive disturbances of Input-to-State Stability (ISS) type.
\end{abstract}

\section{INTRODUCTION}

Lyapunov function based techniques are central in the study of dynamical systems. This is especially true for those having an infinite number of dynamics. These systems are usually modelled by time-delay systems or partial differential equations (PDEs). For the latter family of systems, Lyapunov functions are useful for the analysis of many different types of problems, such as the existence of solutions for the heat equation [1], or the controllability of the semilinear wave equation [2]. The present paper focuses on a class of onedimensional hyperbolic equations like those written as a system of conservation laws. The study of this class of PDEs is crucial when considering a wide range physical networks having an engineering relevance. Among the potential applications we have in mind, there are for instance hydraulic networks (for irrigation or navigation), electric line networks, road traffic networks [3] or gas flow in pipeline networks [4], [5]. The importance of these applications motivates a lot of theoretical questions on hyperbolic systems which for instance pertain to optimal control and controllability as considered in [6], [7], [8].

The stabilizability of such systems is often proved by means of a Lyapunov function as illustrated by the contributions [5], [9], [10] where different control problems are solved for particular hyperbolic equations (see also [11]for the use of Riemann coordinates techniques). For more general nonlinear hyperbolic equations, the knowledge of Lyapunov functions can be useful for the stability analysis of a system of conservation laws (see [12]), or even for the design for these systems of stabilizing boundary controls (see the recent work [13]).

To demonstrate asymptotic stability through the knowledge of a weak Lyapunov function i.e. a Lyapunov function

Christophe Prieur is with Department of Automatic Control, Gipsa-lab, 961 rue de la Houille Blanche, BP 46, 38402 Grenoble Cedex, France, christophe.prieur@gipsa-lab.grenoble-inp.fr; Frédéric Mazenc is with Team INRIA DISCO, CNRS-Supelec, 3 rue Joliot Curie, 91192 Gif-sur-Yvette, France, Frederic.MAZENC@lss.supelec.fr whose derivative, along the trajectories of the system which is considered, is nonpositive, the celebrated Lasalle invariance principle has to be invoked (see e.g. [1], [14], [15]). It requires to state a precompactness property for the solutions, which may be difficult to prove (and is not even always satisfied, as illustrated by the hyperbolic systems considered in [12]). This technical step is not needed when is available a strict Lyapunov function i.e. a Lyapunov function whose derivative, along the trajectories of the system which is considered, is negative definite. Thus designing such a Lyapunov function is a way to overcome this technical difficulty, as done for example in [12]. These remarks motivate the present paper which is devoted to new Lyapunov techniques for the study of stability and robustness properties of Input-toState-Stable (ISS) type for a family of time-varying linear hyperbolic PDEs with disturbances. By first applying the classical technique of construction of Lyapunov functions available in the literature, we will obtain a function which is a weak Lyapunov function for some of the systems we consider, but not for the others. Next, we will transform this function through a strictification approach, which owes a great deal to the one presented in [16], (see also [17], [18]) and obtain that way a strict Lyapunov function which allows us to estimate the robustness of the stability of the systems with respect to the presence of uncertainties and or external disturbances. The function we shall construct is given by an explicit expression.

It is worth mentioning that although the ISS notion is very popular in the area of the dynamical systems of finite dimension (see e.g. the recent survey [19]), or for systems with delay (see for instance [20]), the present work is, to the best of our knowledge, the first one which uses it to characterize a robustness property for hyperbolic PDEs. This work parallels what has been done in [21] where ISS-Lyapunov functions for semilinear time-invariant parabolic PDEs are derived using strictification techniques (see also [22] where ISS properties are compared for a reaction-diffusion system with its finite dimensional counterpart without diffusion). On the other hand, the construction we shall present is significantly disconnected from the one in [21] because the family of systems we will study is very different from the one considered in [21]. In particular the systems studied in [21] are time-invariant whereas the systems considered in the present work are time-varying.

The paper is organized as follows. Basic definitions and notations are introduced in Section II. In Section III the analysis of the robustness of a linear time-varying hyperbolic PDE with uncertainties is carried out by means of the design 
of an ISS Lyapunov function. In Section IV, the main result is illustrated by an example. Concluding remarks in Section $\mathrm{V}$ end the work.

Notation. Throughout the paper, the argument of the functions will be omitted or simplified when no confusion can arise from the context. A function $\alpha$ is said to be of class $\mathcal{K}_{\infty}$ if it is continuous, zero at zero, increasing and unbounded. For any integer $n$, we let $I d$ denote the identity matrix of dimension $n$. Given a continuously differentiable function $A: \mathbb{R}^{n} \rightarrow \mathbb{R}, \frac{\partial A}{\partial \Xi}(\Xi)$ stands for the vector $\left(\frac{\partial A}{\partial \xi_{1}}(\Xi) \ldots . . \frac{\partial A}{\partial \xi_{n}}(\Xi)\right) \in \mathbb{R}^{n}$. The norm induced from the Euclidean inner product of two vectors will be denoted by $|\cdot|$. Given a matrix $A$, its induced matrix norm will be denoted by $\|A\|$, and $\operatorname{Sym}(A)=\frac{1}{2}\left(A+A^{\top}\right)$ stands for the symmetric part of $A$. We denote the set of diagonal positive definite matrices by $\mathcal{D}_{n,+}$. The norm $|\bullet|_{L^{2}(0, L)}$ is defined by: $|\phi|_{L^{2}(0, L)}=\sqrt{\int_{0}^{L}|\phi(z)|^{2} d z}$. Finally, given two topological spaces $X$ and $Y$, we denote by $C^{0}(X ; Y)$ (resp. $\left.C^{1}(X ; Y)\right)$ the set of the continuous (resp. continuously differentiable) functions from $X$ to $Y$. Following [13], we introduce the notation, for all matrices $M \in \mathbb{R}^{n \times n}$,

$$
\rho_{1}(M)=\inf \left\{\left\|\Delta M \Delta^{-1}\right\|, \Delta \in \mathcal{D}_{n,+}\right\} .
$$

\section{BASIC DEFINITIONS AND NOTIONS}

Throughout our work, we will consider partial differential equations of the form

$$
\frac{\partial X}{\partial t}(z, t)+\Lambda(z, t) \frac{\partial X}{\partial z}(z, t)=F(z, t) X(z, t)+\delta(z, t),
$$

where $z \in[0, L], t \in[0,+\infty)$, and $\Lambda(z, t)$ is a diagonal matrix in $\mathbb{R}^{n \times n}$ whose $m$ first diagonal terms are nonnegative and the $n-m$ last terms are nonpositive. We assume that the function $\delta(z, t)$ is a disturbance of class $C^{1}, F(z, t)$ is a periodic function with respect to $t$ of class $C^{1}, \Lambda(z, t)$ is of class $C^{2}$, periodic with respect to $t$ of period $T$.

The boundary conditions are written as

$$
\left(\begin{array}{c}
X_{+}(0, t) \\
X_{-}(L, t)
\end{array}\right)=K\left(\begin{array}{c}
X_{+}(L, t) \\
X_{-}(0, t)
\end{array}\right)
$$

where $X=\left(\begin{array}{c}X_{+} \\ X_{-}\end{array}\right), X_{+} \in \mathbb{R}^{m}, X_{-} \in \mathbb{R}^{n-m}$, and $K \in$ $\mathbb{R}^{n \times n}$ is a constant matrix.

The initial condition is

$$
X(z, 0)=X^{0}(z), \forall z \in(0, L),
$$

where $X^{0}$ is a function in $\left.L^{2}(0, L)\right)$.

When the functions $\Lambda$, and $F$ do not depend on $z$ and $t$, and when $\Lambda$ is invertible, the Cauchy problem (2), (3) and (4) is well-posed (see e.g. [23, Section 2.1 and Section 2.3]). This means that for any initial condition $X^{0}$ in $L^{2}\left((0, L), \mathbb{R}^{n}\right)$, for any $T>0$, and any $\delta$ in $L^{2}((0, L) \times$ $\left.(0, T) ; \mathbb{R}^{n}\right)$, there exists $C(T)>0$ such that a solution $X \in C^{0}\left([0,+\infty) ; L^{2}\left((0, L) ; \mathbb{R}^{n}\right)\right)$ exists, is unique and satisfies, for all $t \in[0, T]$,

$$
|X(., t)|_{L^{2}\left((0, L) ; \mathbb{R}^{n}\right)} \leq C(T)\left|X^{0}\right|_{L^{2}\left((0, L) ; \mathbb{R}^{n}\right)} .
$$

Now we introduce the notions of weak, strict and ISS Lyapunov functions that we consider in this paper (see for instance [15, Def. 3.62] for the notion of Lyapunov functions and [21] for the notion of ISS Lyapunov functions in an infinite dimensional context).

Definition 2.1: Let $\nu: L^{2}(0, L) \times \mathbb{R} \rightarrow \mathbb{R}$ be a continuously differentiable function, periodic with respect to $t$. The function $\nu$ is said to be a weak Lyapunov function for (2) with (3), if there are two functions $\kappa_{S}$ and $\kappa_{M}$ of class $\mathcal{K}_{\infty}$ such that, for all functions $\phi \in L^{2}(0, L)$ and for all $t \in[0,+\infty)$,

$$
\kappa_{S}\left(|\phi|_{L^{2}(0, L)}\right) \leq \nu(\phi, t) \leq \int_{0}^{L} \kappa_{M}(|\phi(z)|) d z
$$

and, in the absence of $\delta$, for all solutions of (2) satisfying (3), and all $t \geq 0$,

$$
\frac{d \nu(X(., t), t)}{d t} \leq 0
$$

The function $\nu$ is said to be a strict Lyapunov function for (2) with (3) if, in the absence of $\delta$, there exits a real number $\lambda_{1}>0$ such that, for all solutions of (2) satisfying (3), and for all $t \geq 0$,

$$
\frac{d \nu(X(., t), t)}{d t} \leq-\lambda_{1} \nu(X(., t), t)
$$

The function $\nu$ is said to be an ISS Lyapunov function for (2) with (3) if there exit a positive real number $\lambda_{1}>0$ and a function $\lambda_{2}$ of class $\mathcal{K}$ such that, for all continuous functions $\delta$, for all solutions of (2) satisfying (3), and for all $t \geq 0$,

$$
\frac{d \nu(X(., t), t)}{d t} \leq-\lambda_{1} \nu(X(., t), t)+\int_{0}^{L} \lambda_{2}(|\delta(z, t)|) d z
$$

Remark 1: 1. For conciseness, we will often use the notation $\dot{\nu}$ instead of $\frac{d \nu(X(., t), t)}{d t}$.

2. Let us recall that, when is known a weak Lyapunov function, asymptotic stability can be often established via the celebrated LaSalle invariance principle applies (see [15, Theorem 3.64] among other references).

3. When a strict Lyapunov function $\nu$ exists for (2) with (3) and $\delta$ is not present, then the value of $\nu$ along the solutions of (2) satisfying (3) exponentially decays to zero and therefore each solution $X(z, t)$ satisfies $\lim _{t \rightarrow+\infty}|X(., t)|_{L^{2}(0, L)}=0$. When in addition, there exists a function $\kappa_{L}$ of class $\mathcal{K}_{\infty}$, such that, for all functions $\phi \in L^{2}(0, L)$ and for all $t \geq 0$,

$$
\nu(\phi, t) \leq \kappa_{L}\left(|\phi|_{L^{2}(0, L)}\right),
$$

then the system (2) is globally asymptotically stable for the topology of the norm $L^{2}$.

4. When the system (2) with (3) admits an ISS Lyapunov function $\nu$, then, one can check through elementary calculations $^{1}$ that, for all solutions of (2) satisfying (3) and for all

\footnotetext{
${ }^{1}$ For instance the next inequality follows from the fact that we have, for all $\kappa$ of class $\mathcal{K}$, and for all positive values $a$ and $b$,

$$
\kappa(a+b) \leq \kappa(2 a)+\kappa(2 b),
$$

and from the fact that the function $\kappa_{S}^{-1}$ is zero at zero and nondecreasing.
} 
instants $t \geq t_{0}$, the inequality

$$
|X(., t)|_{L^{2}(0, L)} \leq \kappa_{S}^{-1}\left(\varphi_{1}\left(t, t_{0}, X\right)\right)+\kappa_{S}^{-1}\left(\varphi_{2}\left(t, t_{0}\right)\right)
$$

with

$$
\varphi_{1}\left(t, t_{0}, X\right)=2 e^{-\lambda_{1}\left(t-t_{0}\right)} \int_{0}^{L} \kappa_{M}\left(\left|X\left(z, t_{0}\right)\right|\right) d z
$$

and

$$
\varphi_{2}\left(t, t_{0}\right)=\frac{2}{\lambda_{1}} \sup _{\ell \in\left[t_{0}, t\right]}\left(\int_{0}^{L} \lambda_{2}(|\delta(z, \ell)|) d z\right)
$$

holds. This inequality is the analogue for the PDEs (2) with (3) of the ISS inequalities for ordinary differential equations. It gives an estimation of the influence of the disturbance $\delta$ on the solutions of the system (2) with (3).

\section{ISS LYAPUNOV FUNCTIONS FOR LINEAR HYPERBOLIC SYSTEMS}

Before stating the main theoretical result of the work, some comments are needed. Since, in the case where the system (2) is such that $m<n$, one can replace $X(z, t)$ by $\left(\begin{array}{c}X_{+}(z, t) \\ X_{-}(L-z, t)\end{array}\right)$ and obtain that way a new PDE whose corresponding diagonal matrix function $\Lambda$ has only nonnegative entries, we may assume without loss of generality that $\Lambda$ is diagonal with nonnegative entries and then the boundary conditions (3) are the boundary condition

$$
X(0, t)=K X(L, t) \text {. }
$$

Next, we recall an important result given in [13] because it sheds light on the problem we consider and, more precisely, on the assumptions we introduce below. If $\Lambda$ is constant, positive, and $\rho_{1}(K)<1$, where the $\rho_{1}$ is the function defined in (1), then the system (2), when $F(z, t)=0$ and $\delta(z, t)=0$ for all $z \in[0, L], t \geq 0$, with the boundary conditions (3) is exponential stable in $H^{2}$-norm. Moreover there exist a diagonal positive definite matrix ${ }^{2} Q \in \mathbb{R}^{n \times n}$ and a positive constant $\varepsilon>0$ such that

$$
\operatorname{Sym}\left(Q \Lambda-K^{\top} Q \Lambda K\right) \geq \varepsilon I d .
$$

Furthermore, following what has been assumed for the parabolic equation in [21], it might seem natural to consider the case where $F(z, t)$ possesses some stability properties. On the other hand, there is no reason to believe that this property is always needed.

These remarks lead us to introduce the following assumption:

Assumption 1: For all $t \geq 0$ and for all $z \in[0, L]$, all the entries of $\Lambda(z, t)$ are nonnegative. There exist a symmetric positive definite matrix $Q$, a real number $\alpha \in(0,1)$, a continuous real-valued function $r$, periodic of period $T>0$ with a positive mean value, i.e. such that the constant

$$
B=\int_{0}^{T} r(m) d m
$$

\footnotetext{
${ }^{2}$ such a matrix $Q$ may be obtained by selecting a diagonal positive matrix $\Delta$ such that $\Delta K \Delta^{-1}<I$. Then selecting $Q=\Delta^{2} \Lambda^{-1}$ and $\varepsilon>0$ sufficiently small we have (8).
}

is positive, satisfying, for all $t \geq 0$ and for all $z \in[0, L]$, the following inequalities:

$$
\begin{gathered}
\operatorname{Sym}\left(\alpha Q \Lambda(L, t)-K^{\top} Q \Lambda(L, t) K\right) \geq 0, \\
\operatorname{Sym}(Q \Lambda(z, t)) \geq r(t) I d, \\
\operatorname{Sym}\left(Q \frac{\partial \Lambda}{\partial z}(z, t)+2 Q F(z, t)\right) \leq 0 .
\end{gathered}
$$

We are ready to state and prove the main result of the paper.

Theorem 1: Assume the system (2) with the boundary conditions (7) satisfies Assumption 1. Let $\mu$ be any real number such that

$$
0<\mu \leq-\frac{1}{L} \ln (\alpha)
$$

Then the function $U: L^{2}(0, L) \times \mathbb{R} \rightarrow \mathbb{R}$ defined, for all $\phi \in L^{2}(0, L)$ and $t \in \mathbb{R}$, by

$$
U(\phi, t)=e^{\frac{1}{T} \int_{t-T}^{t} \int_{\ell}^{t} q(m) d m d \ell} \int_{0}^{L} \phi(z)^{\top} Q \phi(z) e^{-\mu z} d z,
$$

with

$$
q(t)=\frac{\mu}{\|Q\|}\left(r(t)-\frac{B}{2 T}\right),
$$

where $Q$ and $r$ are the matrix and the function in Assumption 1, is an ISS Lyapunov function for the system (2) with (7).

Remark 2: 1 . Assumption 1 does not imply that for all fixed $z \in[0, L]$, the ordinary differential equation $\dot{X}=$ $F(z, t) X$ is stable. In Section IV, we will study an example where this system is exponentially unstable.

2. The fact that $Q$ is symmetric positive definite and all the entries of $\Lambda(z, t)$ are nonnegative does not imply that Sym $(Q \Lambda(z, t))$ is positive definite. That is the reason why we do not assume that $r$ is a nonnegative function.

3. Assumption 1 holds when, in the system $(2), \Lambda(z, t)$ is constant, $F(z, t)$ is constant and diagonal, $\delta(z, t)=0$ for all $z \in[0, L]$ and $t \geq 0$ and the boundary condition (3) satisfies

$$
\operatorname{Sym}\left(Q \Lambda-K^{\top} Q \Lambda K\right) \geq 0, \quad \operatorname{Sym}(Q F) \leq 0
$$

for a suitable symmetric positive definite matrix $Q$. Therefore Theorem 1 generalizes the sufficient conditions of [24] for the exponential stability of linear hyperbolic systems of balance laws to the time-varying case and to the perturbed case.

4. The Lyapunov function $U$ defined in (14) is a timevarying function, periodic of period $T$. In the case where the system is time-invariant, one can chose a constant function $q(t)$, which results with a time-invariant function (14). This function is a quite usual Lyapunov function candidate in the context of the stability analysis of PDEs (see e.g., [13], [25], [26]).

Proof. We begin the proof by showing that the function

$$
V(\phi)=\int_{0}^{L} \phi(z)^{\top} Q \phi(z) e^{-\mu z} d z
$$

is a weak Lyapunov function for the system (2) with (7) when Assumption 1 and (13) are satisfied and $\delta$ is identically equal to zero. 
We note for later use that, for all $\phi \in L^{2}(0, L)$,

$$
\frac{1}{\beta} \int_{0}^{L}|\phi(z)|^{2} d z \leq V(\phi) \leq \beta \int_{0}^{L}|\phi(z)|^{2} d z
$$

with $\beta=\max \left\{\|Q\|, \frac{e^{\mu L}}{\lambda_{Q}}\right\}$, where $\lambda_{Q}$ is the smallest eigenvalue of $Q$. Now, we compute the time-derivative of $V$ along the solutions of (2) with (7):

$$
\begin{aligned}
\dot{V}= & 2 \int_{0}^{L} X(z, t)^{\top} Q \frac{\partial X}{\partial t}(z, t) e^{-\mu z} d z \\
= & 2 \int_{0}^{L} X(z, t)^{\top} Q \\
& \times\left[-\Lambda(z, t) \frac{\partial X}{\partial z}(z, t)+F(z, t) X(z, t)+\delta(z, t)\right] \\
& \times e^{-\mu z} d z \\
= & -R_{1}(X(., t), t)+R_{2}(X(., t), t) \\
& +R_{3}(X(., t), t),
\end{aligned}
$$

with

$$
\begin{gathered}
R_{1}(\phi, t)=2 \int_{0}^{L} \phi(z)^{\top} Q \Lambda(z, t) \frac{\partial \phi}{\partial z}(z) e^{-\mu z} d z \\
R_{2}(\phi, t)=2 \int_{0}^{L} \phi(z)^{\top} Q F(z, t) \phi(z) e^{-\mu z} d z \\
R_{3}(\phi, t)=2 \int_{0}^{L} \phi(z)^{\top} Q \delta(z, t) e^{-\mu z} d z .
\end{gathered}
$$

Now, observe that

$$
\begin{aligned}
R_{1}(\phi, t)= & \int_{0}^{L} \frac{\partial\left(\phi(z)^{\top} Q \Lambda(z, t) \phi(z)\right)}{\partial z} e^{-\mu z} d z \\
& -\int_{0}^{L} \phi(z)^{\top} Q \frac{\partial \Lambda}{\partial z}(z, t) \phi(z) e^{-\mu z} d z .
\end{aligned}
$$

Performing an integration by part on the first integral and using (7), we get

$$
\begin{aligned}
R_{1}(\phi, t)= & \phi(L)^{\top} Q \Lambda(L, t) \phi(L) e^{-\mu L} \\
& -\phi(0)^{\top} Q \Lambda(0, t) \phi(0) \\
& +\mu \int_{Q}^{L} \phi(z)^{\top} Q \Lambda(z, t) \phi(z) e^{-\mu z} d z \\
& -\int_{0}^{L} \phi(z)^{\top} Q \frac{\partial \Lambda}{\partial z}(z, t) \phi(z) e^{-\mu z} d z .
\end{aligned}
$$

Combining (17) and (18), we obtain

$$
\begin{aligned}
\dot{V}= & -X(L, t)^{\top} Q \Lambda(L, t) X(L, t) e^{-\mu L} \\
& +X(0, t)^{\top} Q \Lambda(0, t) X(0, t) \\
& +R_{4}(X(., t), t) \\
& +2 \int_{0}^{L} X(z, t)^{\top} Q \delta(z, t) e^{-\mu z} d z,
\end{aligned}
$$

with

$$
\begin{aligned}
R_{4}(\phi, t)= & -\mu \int_{Q}^{L} \phi(z)^{\top} Q \Lambda(z, t) \phi(z) e^{-\mu z} d z \\
& +\int_{0}^{L} \phi(z)^{\top} Q \frac{\partial \Lambda}{\partial z}(z, t) \phi(z) e^{-\mu z} d z \\
& +2 \int_{0}^{L} \phi(z)^{\top} Q F(z, t) \phi(z) e^{-\mu z} d z .
\end{aligned}
$$

Using (7), we obtain

$$
\begin{aligned}
\dot{V}= & -X(L, t)^{\top} Q \Lambda(L, t) X(L, t) e^{-\mu L} \\
& +X(L, t)^{\top} K^{\top} Q \Lambda(L, t) K X(L, t) \\
& +R_{4}(X(., t), t) \\
& +2 \int_{0}^{L} X(z, t)^{\top} Q \delta(z, t) e^{-\mu z} d z .
\end{aligned}
$$

By grouping the terms and using the notation

$$
N(t)=K^{\top} Q \Lambda(L, t) K
$$

we obtain

$$
\begin{aligned}
\dot{V}= & X(L, t)^{\top}\left[N(t)-e^{-\mu L} Q \Lambda(L, t)\right] X(L, t) \\
& +R_{4}(X(., t), t) \\
& +2 \int_{0}^{L} X(z, t)^{\top} Q \delta(z, t) e^{-\mu z} d z .
\end{aligned}
$$

Grouping the terms in $R_{4}(X(., t), t)$, we obtain

$$
\begin{aligned}
\dot{V}= & X(L, t)^{\top}\left[N(t)-e^{-\mu L} Q \Lambda(L, t)\right] X(L, t) \\
& -\int_{0}^{L} X(z, t)^{\top} Q M(z, t) X(z, t) e^{-\mu z} d z \\
& +2 \int_{0}^{L} X(z, t)^{\top} Q \delta(z, t) e^{-\mu z} d z
\end{aligned}
$$

with

$$
M(z, t)=\mu \Lambda(z, t)-\frac{\partial \Lambda}{\partial z}(z, t)-2 F(z, t) .
$$

The inequalities (10) and (13) imply that

$$
\begin{aligned}
\dot{V} \leq & -\int_{0}^{L} X(z, t)^{\top} Q M(z, t) X(z, t) e^{-\mu z} d z \\
& +2 \int_{0}^{L} X(z, t)^{\top} Q \delta(z, t) e^{-\mu z} d z .
\end{aligned}
$$

It follows from (12) that

$$
\begin{aligned}
\dot{V} \leq & -\mu \int_{0}^{L} X(z, t)^{\top} Q \Lambda(z, t) X(z, t) e^{-\mu z} d z \\
& +2 \int_{0}^{L} X(z, t)^{\top} Q \delta(z, t) e^{-\mu z} d z .
\end{aligned}
$$

Using (11), we deduce that

$$
\begin{aligned}
\dot{V} \leq & -\mu r(t) \int_{0}^{L}|X(z, t)|^{2} e^{-\mu z} d z \\
& +2 \int_{0}^{L} X(z, t)^{\top} Q \delta(z, t) e^{-\mu z} d z .
\end{aligned}
$$

From (16), we deduce that

$$
\begin{aligned}
\dot{V} \leq & -\frac{\mu}{\|Q\|} r(t) V(X(., t)) \\
& +2\|Q\| \int_{0}^{L}|X(z, t) \| \delta(z, t)| e^{-\mu z} d z .
\end{aligned}
$$

It follows that, for all $\kappa>0$,

$$
\begin{aligned}
\dot{V} \leq & -\frac{\mu}{\|Q\|} r(t) V(X(., t)) \\
& +2\|Q\| \kappa \int_{0}^{L}|X(z, t)|^{2} e^{-\mu z} d z \\
& +\frac{\|Q\|}{2 \kappa} \int_{0}^{L}|\delta(z, t)|^{2} e^{-\mu z} d z \\
\leq & -q_{\kappa}(t) V(X(., t))+\frac{\|Q\|}{2 \kappa} \int_{0}^{L}|\delta(z, t)|^{2} d z,
\end{aligned}
$$


with $q_{\kappa}(t)=\frac{\mu}{\|Q\|} r(t)-\frac{2\|Q\| \kappa}{\lambda_{Q}}$.

The inequality (19) implies that when $r(t)$ is nonnegative and $\delta$ is identically equal to zero, the function $V$ is a weak Lyapunov function for the system (2) with the initial conditions (7). However, we did not assume that the function $r$ is nonnegative and we aim at establishing that the system is ISS with respect to $\delta$. This leads us to apply a strictification technique which transforms $V$ into a strict Lyapunov function. The technique of [16, Chapter 11] leads us to consider the time-varying candidate Lyapunov function

$$
U_{\kappa}(t, \phi)=e^{s_{\kappa}(t)} V(\phi),
$$

with $s_{\kappa}(t)=\frac{1}{T} \int_{t-T}^{t} \int_{\ell}^{t} q_{\kappa}(m) d m d \ell$. Through elementary calculations, one can prove that the time-derivative of $U_{\kappa}$ along the solutions of (2) with the initial conditions (7) satisfies:

$$
\begin{aligned}
\dot{U}_{\kappa} \leq & -e^{s_{\kappa}(t)} q_{\kappa}(t) V(X(., t)) \\
& +\frac{\|Q\|}{2 \kappa} e^{s_{\kappa}(t)} \int_{0}^{L}|\delta(z, t)|^{2} d z \\
& +e^{s_{\kappa}(t)}\left[q_{\kappa}(t)-\frac{1}{T} \int_{t-T}^{t} q_{\kappa}(m) d m\right] V(X(., t)) \\
\leq & \frac{\|Q\|}{2 \kappa} e^{s_{\kappa}(t)} \int_{0}^{L}|\delta(z, t)|^{2} d z \\
& -e^{s_{\kappa}(t)} \frac{1}{T} \int_{t-T}^{t} q_{\kappa}(m) d m V(X(., t)) .
\end{aligned}
$$

Since $r$ is periodic of period $T$, we have

$$
\begin{aligned}
\int_{t-T}^{t} q_{\kappa}(m) d m & =\int_{t-T}^{t} \frac{\mu}{\|Q\|} r(m) d m-\frac{2 T\|Q\| \kappa}{\lambda_{Q}} \\
& =\frac{\mu}{\|Q\|} B-\frac{2 T\|Q\| \kappa}{\lambda_{Q}},
\end{aligned}
$$

where $B$ is the constant defined in (9). We deduce that the value

$$
\kappa=\frac{\mu B \lambda_{Q}}{4 T\|Q\|^{2}},
$$

which is positive because $B$ is positive, gives

$$
\begin{aligned}
\dot{U} \leq & -\frac{\mu}{2\|Q\|} B U(X(., t), t) \\
& +\frac{\|Q\|}{2 \kappa} e^{s_{\kappa}(t)} \int_{0}^{L}|\delta(z, t)|^{2} d z \\
\leq & -\frac{\mu}{2\|Q\|} B U(X(., t), t)+c_{1} \int_{0}^{L}|\delta(z, t)|^{2} d z,
\end{aligned}
$$

with $c_{1}=T \frac{\|Q\|^{3}}{\mu B \lambda_{Q}} e^{T \frac{\mu r_{M}}{\|Q\|}}, r_{M}=\sup _{\{m \in[0, T]\}}\{r(m)\}$ and $U=$ $U_{\kappa}$ for $\kappa$ defined in (20). Moreover, (16) ensures that, for all $t \in \mathbb{R}$ and $\phi \in L^{2}(0, L)$,

$$
c_{2} \int_{0}^{L}|\phi(z)|^{2} d z \leq U(t, \phi) \leq c_{3} \int_{0}^{L}|\phi(z)|^{2} d z
$$

with $c_{2}=\frac{1}{\beta} e^{\frac{\mu}{4\|Q\|}\left(2 T r_{S}-B\right)}, r_{S}=\inf _{\{m \in[0, T]\}}\{r(m)\}$ and $c_{3}=\exp \left(\frac{T \mu r_{M}}{\|Q\|}\right) \beta$. Therefore inequalities of the type (5) are satisfied. We deduce that $U$ is an ISS Lyapunov function, as introduced in Definition 2.1.

This concludes the proof of Theorem 1 .

\section{BENCHMARK EXAMPLE}

In this section we consider the system (2) and the boundary condition (3) with the following data, for all $z$ in $(0,1)$ and for all $t \geq 0$,

$$
\begin{aligned}
& X(z, t) \in \mathbb{R}, L=1, \\
& \Lambda(z, t)=\cos ^{2}(t)+1-z, F(z, t)=\frac{\sin ^{2}(t)}{5} \\
& K=\frac{1}{2} .
\end{aligned}
$$

A remarkable feature of this system if that the system $\dot{\xi}=$ $F(z, t) \xi$, which rewrites as $\dot{\xi}=\frac{\sin ^{2}(t)}{5} \xi$ is exponentially unstable. Now, we show that Theorem 1 applies to it. Let $Q$ be any positive real number. Then we have, for all $t \geq 0$, $N(t)=\frac{Q}{4} \cos ^{2}(t)$ and $Q \Lambda(L, t)=Q \cos ^{2}(t)$ and thus (10) is satisfied with $\alpha=\frac{1}{4}$. It is clear that (11) holds with $r(t)=$ $\cos ^{2}(t)$. Finally, for all $z \in[0, L], t \geq 0$,

$$
\begin{aligned}
\operatorname{Sym}\left(Q \frac{\partial \Lambda}{\partial z}(z, t)+2 Q F(z, t)\right) & =-Q+2 Q \frac{\sin ^{2}(t)}{5} \\
& \leq-\frac{3 Q}{5} .
\end{aligned}
$$

Therefore (12) holds and Assumption 1 is satisfied. We conclude that Theorem 1 applies. It follows that the system

$$
\frac{\partial X}{\partial t}(z, t)+\left(\cos ^{2}(t)+1-z\right) \frac{\partial X}{\partial z}(z, t)=\frac{\sin ^{2}(t)}{5} X(z, t)
$$

for all $z$ in $(0,1)$ and for all $t \geq 0$, with the boundary condition $X(0, t)=\frac{1}{2} X(1, t)$ is asymptotically stable and the function (14) is an ISS Lyapunov function (with respect to $\delta$ ).

\section{CONCLUSIONS}

For time-varying hyperbolic PDEs, we have designed ISS-Lyapunov functions. These functions are time-varying and periodic. They make it possible to derive robustness properties of the ISS type.

This work leaves many questions open. The problem of designing ISS Lyapunov functions for nonlinear hyperbolic equations will be considered in future works, possibly with the help of Lyapunov functions considered in [13]. In addition, it would be of interest to use an experimental channel to validate experimentally the prediction of the offset that is inferred from the ISS-Lyapunov function in a similar way as what is done in [27].

\section{REFERENCES}

[1] T. Cazenave and A. Haraux, An introduction to semilinear evolution equations. Oxford University Press, 1998.

[2] J.-M. Coron and E. Trélat, "Global steady-state stabilization and controllability of 1D semilinear wave equations," Commun. Contemp. Math., vol. 8, no. 4, pp. 535-567, 2006.

[3] B. Haut and G. Bastin, "A second order model of road junctions in fluid models of traffic networks," Networks and Heterogeneous Media, vol. 2, no. 2, pp. 227-253, june 2007.

[4] G. Bastin, J.-M. Coron, and B. d'Andréa Novel, "Using hyperbolic systems of balance laws for modeling, control and stability analysis of physical networks," in Lecture notes for the Pre-Congress Workshop on Complex Embedded and Networked Control Systems. Seoul, Korea: 17th IFAC World Congress, 2008.

[5] M. Dick, M. Gugat, and G. Leugering, "Classical solutions and feedback stabilization for the gas flow in a sequence of pipes," Networks and Heterogeneous Media, vol. 5, no. 4, pp. 691-709, 2010. 
[6] M. Gugat, M. Herty, A. Klar, and G. Leugering, "Optimal control for traffic flow networks," Journal of Optimization Theory and Applications, vol. 126, no. 3, pp. 589-616, 2005.

[7] R. M. Colombo, G. Guerra, M. Herty, and V. Schleper, "Optimal control in networks of pipes and canals," SIAM Journal on Control and Optimization, vol. 48, pp. 2032-2050, 2009.

[8] M. Gugat and G. Leugering, "Global boundary controllability of the Saint-Venant system for sloped canals with friction," Annales de l'Institut Henri Poincare (C) Non Linear Analysis, vol. 26, no. 1, pp. 257-270, 2009.

[9] M. Krstic and A. Smyshlyaev, "Backstepping boundary control for first order hyperbolic pdes and application to systems with actuator and sensor delays," in 46th IEEE Conference on Decision and Control $(C D C)$, New Orleans, LA, USA, 2007, pp. 225-230.

[10] _ - "Backstepping boundary control for first-order hyperbolic pdes and application to systems with actuator and sensor delays," Systems \& Control Letters, vol. 57, pp. 750-758, 2008.

[11] C. Prieur, J. Winkin, and G. Bastin, "Robust boundary control of systems of conservation laws," Mathematics of Control, Signals, and Systems, vol. 20, no. 2, pp. 173-197, 2008.

[12] J.-M. Coron, B. d'Andréa Novel, and G. Bastin, "A strict Lyapunov function for boundary control of hyperbolic systems of conservation laws," IEEE Transactions on Automatic Control, vol. 52, no. 1, pp. 2-11, 2007.

[13] J.-M. Coron, G. Bastin, and B. d'Andréa Novel, "Dissipative boundary conditions for one-dimensional nonlinear hyperbolic systems," SIAM Journal on Control and Optimization, vol. 47, no. 3, pp. 1460-1498, 2008.

[14] M. Slemrod, "A note on complete controllability and stabilizability for linear control systems in Hilbert space," SIAM Journal on Control, vol. 12, pp. 500-508, 1974.

[15] Z.-H. Luo, B.-Z. Guo, and O. Morgul, Stability and stabilization of infinite dimensional systems and applications, ser. Communications and Control Engineering. New York: Springer-Verlag, 1999.

[16] M. Malisoff and F. Mazenc, Constructions of Strict Lyapunov Functions, ser. Communications and Control Engineering Series. London, UK: Springer, 2009.
[17] F. Mazenc and D. Nesic, "Lyapunov functions for time-varying systems satisfying generalized conditions of Matrosov theorem," Mathematics of Control, Signals, and Systems, vol. 19, pp. 151-182, 2007.

[18] F. Mazenc, M. Malisoff, and O. Bernard, "A simplified design for stric Lyapunov functions under Matrosov conditions," IEEE Transactions on Automatic Control, vol. 54, no. 1, pp. 177-183, jan. 2009.

[19] E. Sontag, "Input to state stability: Basic concepts and results," in Nonlinear and Optimal Control Theory. Berlin: Springer-Verlag, 2007, pp. 163-220.

[20] F. Mazenc, M. Malisoff, and Z. Lin, "Further results on input-to-state stability for nonlinear systems with delayed feedbacks," Automatica, vol. 44, no. 9, pp. 2415-2421, 2008.

[21] F. Mazenc and C. Prieur, "Strict Lyapunov functions for semilinear parabolic partial differential equations," Mathematical Control and Related Fields, vol. 1, no. 2, pp. 231-250, 2011.

[22] S. Dashkovskiy and A. Mironchenko, "On the uniform input-to-state stability of reaction-diffusion systems," in 49th IEEE Conference on Decision and Control (CDC), Atlanta, GA, USA, 2010, pp. 65476552.

[23] J.-M. Coron, Control and nonlinearity, ser. Mathematical Surveys and Monographs. Providence, RI: American Mathematical Society, 2007, vol. 136.

[24] A. Diagne, G. Bastin, and J.-M. Coron, "Lyapunov exponential stability of linear hyperbolic systems of balance laws," CESAME, UCL, Louvain-la-Neuve, Belgium, Tech. Rep., 2010.

[25] J.-M. Coron, "On the null asymptotic stabilization of the twodimensional incompressible Euler equations in a simply connected domain," SIAM Journal on Control and Optimization, vol. 37, no. 6, pp. 1874-1896, 1999.

[26] Cheng-Zhong $\mathrm{Xu}$ and G. Sallet, "Exponential stability and transfer functions of processes governed by symmetric hyperbolic systems," ESAIM Control, Optimisation and Calculus of Variations, vol. 7, pp. 421-442, 2002.

[27] V. Dos Santos and C. Prieur, "Boundary control of open channels with numerical and experimental validations," IEEE Trans. Control Syst. Tech., vol. 16, no. 6, pp. 1252-1264, 2008. 